\title{
A novel uniform asymptotic solution for reflection of a Gaussian beam at a dielectric interface
}

\author{
Dinh T. Quang, Keiji Goto, Toru Kawano, and Toyohiko Ishihara ${ }^{\text {a) }}$ \\ Department of Communication Engineering, National Defense Academy \\ 1-10-20 Hashirimizu, Yokosuka, 239-8686, Japan \\ a) ishihara@cc.nda.ac.jp
}

\begin{abstract}
We have derived a novel uniform asymptotic solution for reflection of the Gaussian beam that is incident on a dielectric interface from a denser medium to a rarer one. The validity of the novel uniform asymptotic solution has been confirmed by comparing with the reference solution. It has been shown that the transition beam plays an important role to connect two different solutions smoothly through the transition region. It becomes apparent that, near the critical angle of the total reflection, the Gaussian beam is displaced to either direction by the interference between the geometrically reflected beam and the lateral beam.
\end{abstract}

Keywords: asymptotic solution, reflection, lateral beam, Gaussian beam, transition beam, dielectric interface

Classification: Electromagnetic theory

\section{References}

[1] H. K. V. Lotsch, "Beam displacement at total reflection: The GoosHänchen effect I," Optik, vol. 32, no. 2, pp. 116-137, April 1970.

[2] B. R. Horowitz and T. Tamir, "Unified theory of total reflection phenomena at a dielectric interface," Appl. Phys., vol. 1, pp. 31-38, 1973.

[3] S. Kozaki and H. Sakurai, "Characteristics of a Gaussian beam at a dielectric interface," J. Opt Soc. Am., vol. 68, no. 4, pp. 508-514, April 1978.

[4] T. Tamir, "Nonspecular phenomena in beam fields reflected by multilayered media," J. Opt Soc. Am. A., vol. 3, no. 4, pp. 558-565, April 1986.

[5] S. Zhang and C. Fan, "Nonspecular phenomena on Gaussian beam reflection at dielectric interfaces," J. Opt. Soc. Am. A, vol. 5, no. 9, pp. 14071409, Sept. 1988.

[6] M. Hashimoto, "The law of total reflection in geometrical optics based on wave-normal rays," IEICE Trans. Electron., vol. J72-C-I, no. 3, pp. 131138, March 1989.

[7] A. Ishimaru, J. R. Thomas, and S. Jaruwatanadilok, "Electromagnetic waves over half-space metamaterials of arbitrary permittivity and permeability," IEEE Trans. Antennas Propag., vol. 53, no. 3, pp. 915-921, March 2005. 
[8] Q. Li and R. J. Vernon, "Theoretical and experimental investigation of Gaussian beam transmission and reflection by a dielectric slab at $110 \mathrm{GHz}, "$ IEEE Trans. Antennas Propag., vol. 54, no. 11, pp. 34493454, Nov. 2006.

[9] L. M. Brekhovskikh, Waves in Layered Media, 2nd ed., chaps.1 and 4, Academic Press, New York, 1980.

[10] T. Ishihara and Y. Miyagawa, "A uniform asymptotic analysis for the scattered electromagnetic field on the plane dielectric interface excited by a vector point source," IEICE Trans. Electron., vol. J82-C-I, no. 2, pp. 62-73, Feb. 1999.

[11] H. Yamada, T. Kawano, K. Goto, and T. Ishihara, "A uniform asymptotic analysis of a Gaussian beam scattered by a dielectric interface," The papers of Technical Meeting on Electromagnetic Theory, IEE Japan, EMT-01-40, pp. 25-30, May 2007.

[12] D. T. Quang, K. Goto, T. Kawano, and T. Ishihara, "Asymptotic analysis of transmitted wave in transition region through a plane dielectric interface," The papers of Technical Meeting on Electromagnetic Theory, IEE Japan, EMT-10-129, pp. 153-157, Nov. 2010.

[13] L. B. Felsen and N. Marcuvitz, eds., Radiation and Scattering of Waves, chap. 4, IEEE Press, (Classic Reissue), New Jersey, USA, 1994.

[14] E. T. Whittaker and G. N. Watson, A Course of Modern Analysis, 4th eds., pp. 347-351, Syndics of Cambridge University Press, London, 1927 (Reprinted 1973).

\section{Introduction}

Past studies on the reflection of a Gaussian beam that is incident from a denser dielectric medium to a rarer one have shown that the Gaussian beam reflected by a single medium interface do not exactly follow the path of geometrical-optics $[1,2,3,4,5,6,7,8]$. Many theoretical studies are concerned with the investigation of the nonspecular effects in the Gaussian beam reflection on the dielectric interface $[1,2,3,4,5,6,8]$.

The studies on the asymptotic analysis methods for the reflected Gaussian beam fields applicable uniformly through the transition region are not enough until today $[2,9,10,11,12]$. Horowitz and Tamir [2] have investigated the basic wave mechanism, including both the Goos-Hänchen shift and the weak radiance that accompanies the reflected beam. The integral representation and its asymptotic solution for the Gaussian beam reflection by Horowitz and Tamir [2] can be applied in the limited case when the Gaussian beam is incident on the dielectric interface nearly from the normal direction to the interface.

In this paper, we shall investigate the reflection of a Gaussian beam that is incident on the interface from a denser dielectric medium to a rarer one at an arbitrary angle $[9,10,11,12]$. We will derive a novel uniform asymptotic solution for reflection of the Gaussian beam consisting of the geometrically reflected beam, the lateral beam, and the transition beam. The validity and the physical phenomena of the novel uniform asymptotic solution for the Gaussian beam reflection are confirmed by comparing with the reference 
solution calculated numerically.

\section{Uniform asymptotic solution for Gaussian beam reflection}

\subsection{Formulation and integral representations}

Fig. 1 shows the schematic figure for reflection of a Gaussian beam incident on a plane dielectric interface from a denser dielectric medium $\left(\varepsilon_{1}, \mu_{0}\right)$ to a rarer one $\left(\varepsilon_{2}, \mu_{0}\right)$, the Cartesian coordinate system $(x, y, z)$, the incident beam coordinate system $\left(x_{i}, z_{i}\right)$, and the reflected beam coordinate system $\left(x_{r}, z_{r}\right)$. The relative refractive index $n$ defined by $n=\sqrt{\varepsilon_{2} / \varepsilon_{1}}$ satisfies $n<1$.

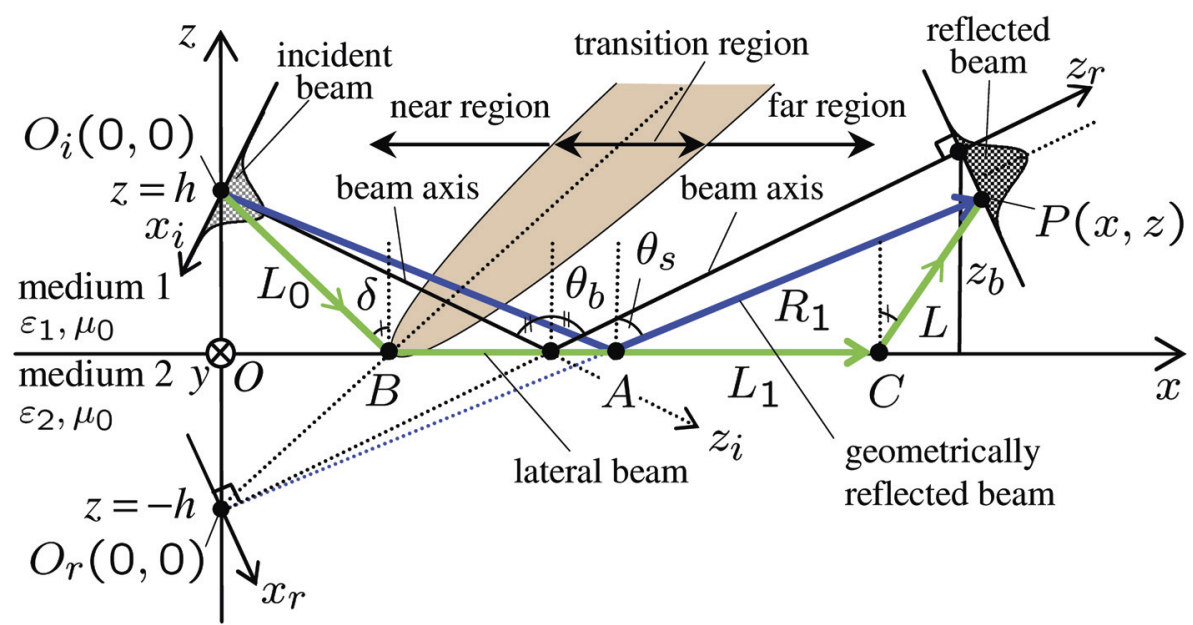

Fig. 1. Reflection of Gaussian beam by a plane dielectric interface and coordinate systems.

We assume that the $y$-component of the incident Gaussian beam $U_{y}^{i}\left(x_{i}, 0\right)$ along the aperture plane $z_{i}=0$ is given by $[2,3,11]$

$$
U_{y}^{i}\left(x_{i}, 0\right)=\frac{1}{\sqrt{\pi} W} \exp \left\{-\left(x_{i} / W\right)^{2}\right\}
$$

in the incident beam coordinate system $\left(x_{i}, z_{i}\right)$. Here $2 W$ denotes the beam width, and $U_{y}^{i}\left(x_{i}, 0\right)=H_{y}^{i}\left(x_{i}, 0\right)$ for the TM case and $U_{y}^{i}\left(x_{i}, 0\right)=E_{y}^{i}\left(x_{i}, 0\right)$ for the TE case. Then one may obtain the reflected Gaussian beam representation observed at $P(x, z)$ in $(x, z)$ coordinate system as follows $[2,11,12]$ :

$$
\begin{gathered}
U_{y}^{r}(x, z)=\frac{k_{1}}{2 \pi} \int_{P_{\theta}} \Gamma(\theta) B\left(\theta, \theta_{b}\right) e^{i k_{1} R_{1} q(\theta)} d \theta \\
B\left(\theta, \theta_{b}\right)=e^{-\left\{k_{1} W \sin \left(\theta-\theta_{b}\right) / 2\right\}^{2}} \cos \left(\theta-\theta_{b}\right) \\
\Gamma(\theta)=\frac{m \cos \theta-\sqrt{n^{2}-\sin ^{2} \theta}}{m \cos \theta+\sqrt{n^{2}-\sin ^{2} \theta}}, \quad q(\theta)=\cos \left(\theta-\theta_{s}\right)
\end{gathered}
$$

where $m=n^{2}$ in the TM case and $m=1$ in the TE case. The notations $k_{1}, R_{1}\left(=\sqrt{x^{2}+(h+z)^{2}}\right), \theta_{b}$, and $\theta_{s}$ denote, respectively, the wavenumber in the upper medium 1 , the propagation distance along $Q_{i}(0,0) \rightarrow A \rightarrow$ 
$P(x, z)$, the incident angle of the beam axis, and the incident angle of the geometrically reflected beam (see Fig. 1). In Fig. 2 (a), the integration path $P_{\theta}$ and the branch point singularities $\pm \delta\left(\delta=\sin ^{-1} n\right)$ associated with the branch cuts are shown in the complex $\theta$-plane.

To obtain a novel uniform asymptotic solution, one may express $\Gamma(\theta)$ in (4) as follows:

$$
\begin{gathered}
\Gamma(\theta)=\Gamma\left(\theta_{s}\right)+\Gamma^{s}(\theta), \quad \Gamma^{s}(\theta)=\bar{\Gamma}^{s}(\theta)+\Gamma^{(2)}(\theta) \sqrt{\sin (\delta-\theta)} \\
\bar{\Gamma}^{s}(\theta)=\Gamma^{(1)}(\theta)-\Gamma^{(1)}\left(\theta_{s}\right)-\Gamma^{(2)}\left(\theta_{s}\right) \sqrt{\sin \left(\delta-\theta_{s}\right)} \\
\Gamma^{(1)}(\theta)=\frac{m^{2} \cos ^{2} \theta-\left(\sin ^{2} \theta-n^{2}\right)}{m^{2} \cos ^{2} \theta+\left(\sin ^{2} \theta-n^{2}\right)} \\
\Gamma^{(2)}(\theta)=\frac{-2 m \cos \theta}{m^{2} \cos ^{2} \theta+\left(\sin ^{2} \theta-n^{2}\right)} \sqrt{\sin (\delta+\theta)}
\end{gathered}
$$

where $\theta_{s}$ used in the above equations is the saddle point of the integrand in (2) determined from $(d / d(\theta)) q(\theta)=0$. Now substituting (5) associated with $(5 a) \sim(5 c)$ into $(2)$ and suppressing the subscript " $y$ " and the coordinate system " $(x, z)$ " in the notations, one may obtain

$$
\begin{gathered}
U^{r}=U^{g o}+U^{s} \\
U^{g o}=\frac{k_{1}}{2 \pi} \Gamma\left(\theta_{s}\right) \int_{P_{\theta}} B\left(\theta, \theta_{b}\right) e^{i k_{1} R_{1} q(\theta)} d \theta
\end{gathered}
$$

where the scattered beam field $U^{s}$ in (6) is expressed by the summation of the scattered beam integrals $U^{s 1}$ and $U^{s 2}$ as follows.

$$
\begin{gathered}
U^{s}=U^{s 1}+U^{s 2} \\
U^{s 1}=\frac{k_{1}}{2 \pi} \int_{P_{\theta}} \bar{\Gamma}^{s}(\theta) B\left(\theta, \theta_{b}\right) e^{i k_{1} R_{1} q(\theta)} d \theta \\
U^{s 2}=\frac{k_{1}}{2 \pi} \int_{P_{\theta}} \Gamma^{(2)}(\theta) \sqrt{\sin (\delta-\theta)} B\left(\theta, \theta_{b}\right) e^{i k_{1} R_{1} q(\theta)} d \theta
\end{gathered}
$$

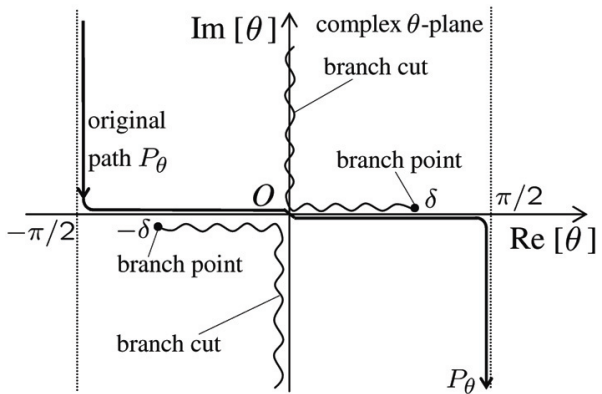

(a) Original integration path $P_{\theta}$ and branch points $\pm \delta$ associated with branch cuts.

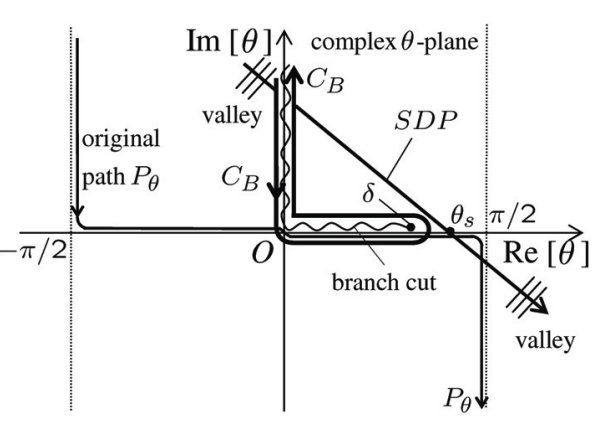

(b) Steepest descent path SDP and integration path $C_{B}$ around branch cut.

Fig. 2. Integration paths in complex $\theta$-plane. 


\subsection{Novel asymptotic solutions for reflected Gaussian beam}

The geometrically reflected beam integral $U^{g o}$ in (7) and the scattered beam integral $U^{s 1}$ in (9) can be evaluated asymptotically by applying the isolated saddle point technique $[11,13]$ for large $k_{1} R_{1}$. While the integral $U^{s 2}$ in (10) is evaluated by deforming the integration path $P_{\theta}$ into the steepest descent path $S D P$ passing through the saddle point $\theta_{s}$ (see Fig. $2(\mathrm{~b})$ ). The branch cut contribution $U^{B}$ arises when the saddle point $\theta_{s}$ is larger than the branch point $\delta$. Thus $U^{s 2}$ can be represented by [11]

$$
U^{s 2}=U^{S D P}+u\left(\theta_{s}-\delta\right) U^{B}
$$

The integrals for $U^{S D P}$ and $U^{B}$ are obtained from (10) by replacing the integration path $P_{\theta}$ by $S D P$ and $C_{B}$, respectively, where $C_{B}$ denotes the integration path around the branch cut (see Fig. $2(\mathrm{~b})$ ). In (11), $u\left(\theta_{s}-\delta\right)$ is 1 for $\theta_{s}>\delta$ and 0 for $\theta_{s}<\delta$.

When the geometrically reflected beam solution for $U^{g o}$ in (7) and the scattered beam solution for $U^{s}$ in (8) associated with the asymptotic solutions for $U^{s 1}$ in (9) and $U^{s 2}$ in (11) are substituted into $U^{r}$ in (6) and the solutions $U^{s 1}$ and $U^{S D P}$ are combined to produce the transition beam $U^{\text {tran }}$, one may obtain a novel uniform asymptotic solution as follows.

$$
U^{r}=U^{g o}+u\left(\theta_{s}-\delta\right) U^{B}+U^{\text {tran }}
$$

The geometrically reflected beam $U^{g o}$, the lateral beam $U^{B}$, and the transition beam $U^{\text {tran }}$ are given, respectively, by

$$
\begin{gathered}
U^{g o}=\Gamma\left(\theta_{s}\right) \sqrt{\frac{k_{1}}{2 \pi R_{1}}} B\left(\theta_{s}, \theta_{b}\right) e^{i k_{1} R_{1}-i \pi / 4} \\
U^{B}=\frac{k_{1}}{m} B\left(\delta, \theta_{b}\right) \frac{D_{-3 / 2}(\eta+i \eta) e^{i 5 \pi / 8}}{\left\{k_{1} \cos \left(\theta_{s}-\delta\right)\right\}^{3 / 4} e^{-i \eta^{2} / 2}} \sqrt{\frac{2 n}{\pi \cos \delta}} e^{i k_{1}\left(L_{0}+L\right)+i k_{2} L_{1}} \\
U^{\text {tran }}=i \Gamma^{(2)}\left(\theta_{s}\right) \sqrt{\sin \left(\theta_{s}-\delta\right)} B\left(\theta_{s}, \theta_{b}\right)\left\{\frac{D_{1 / 2}(\eta+i \eta)}{2^{1 / 4} \eta^{1 / 2} e^{-i \pi / 8} e^{i \eta^{2} / 2}}-1\right\} \\
\cdot \sqrt{\frac{k_{1}}{2 \pi R_{1}}} e^{i k_{1} R_{1}-i \pi / 4}, \quad \eta=\sqrt{2 k_{1} R_{1}} \sin \frac{\theta_{s}-\delta}{2}
\end{gathered}
$$

where $D_{-3 / 2}(z)$ and $D_{1 / 2}(z)$ are the parabolic cylinder functions $[2,9,10,11$, 14], and $k_{2}$ is the wavenumber in the medium 2 . The geometrically reflected beam $U^{g o}$ in (13) and the lateral beam $U^{B}$ in (14) are shown in Fig. 1.

When the observation point $P(x, z)$ is located in the region satisfying $\theta_{s}<\delta$, the transition beam $U^{\operatorname{tran}}$ in (12) for $\theta_{s}<\delta$ can be obtained directly from (15) by substituting $-i \sqrt{\sin \left(\delta-\theta_{s}\right)}$ for $\sqrt{\sin \left(\theta_{s}-\delta\right)}$ and $\zeta$ for $\eta$ in (15). Here $\zeta$ is given by $\zeta=\sqrt{2 k_{1} R_{1}} \sin \left\{\left(\delta-\theta_{s}\right) / 2\right\}$.

\section{Numerical results and discussions}

In Fig. 3 (a) and Fig. 3 (b), we have calculated the TM type (i.e., $m=n^{2}$ ) Gaussian beams reflected on the dielectric interface for two incident beam 
angles $\theta_{b}=60^{\circ}$, which is equal to the critical angle $\delta$ of the total reflection, and $\theta_{b}=60.7^{\circ}$, respectively. The geometrically reflected beam solution $U^{g o}\left(=H^{g o}\right)$, the lateral beam solution $U^{B}\left(=H^{B}\right)$, and the transition beam solution $U^{\text {tran }}\left(=H^{\text {tran }}\right)$ are shown, respectively, by the blue curve $(-)$, the green curve $(-)$, and the brown curve $(-)$. The total Gaussian beam solution $H^{r}$ calculated from (12) is shown by the red curve ( - ) and is compared with the reference solution ( $\circ \circ \circ$ : open circles) calculated numerically from the integral representation in (2).

It is clarified that the total Gaussian beam solution ( - ) calculated from (12) agrees excellently with the reference solution ( $\circ \circ \circ)$ in all regions including the transition region shown by the sign $\leftrightarrow$ in Fig. 3 (a) and 3 (b). Note that, in (12), the solution in the near region where $\theta_{s}<\delta$ is different from the solution in the far region where $\theta_{s}>\delta$. It is shown that the transition beam ( - ) plays an important role to connect two different solutions in the near region and in the far region smoothly through the transition region (see Fig. 3 (a), (b) and also Fig. 1).

It is very interesting to observe that the point where the total beam field ( — ) takes the maximum value is shifted to the right direction (to the left direction) from the beam axis where $x_{r}=0$ in Fig. 3 (a) (in Fig. $3(\mathrm{~b})$ ). It becomes apparent that, near the critical angle of the total reflection, the Gaussian beam is displaced to either direction due to the interference between the geometrically reflected beam and the lateral beam [11].

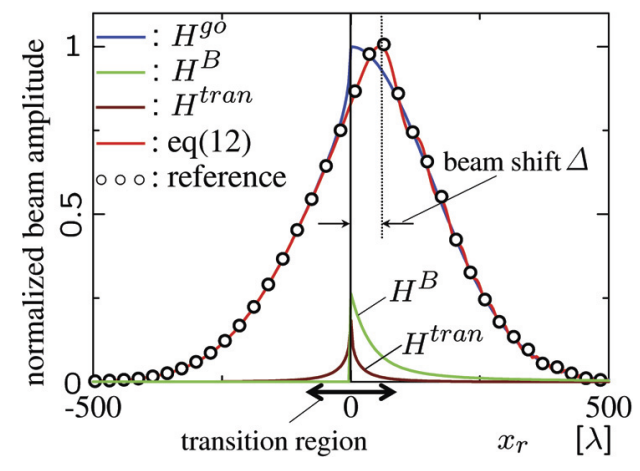

(a) $\theta_{b}=60^{\circ}$ (critical angle)

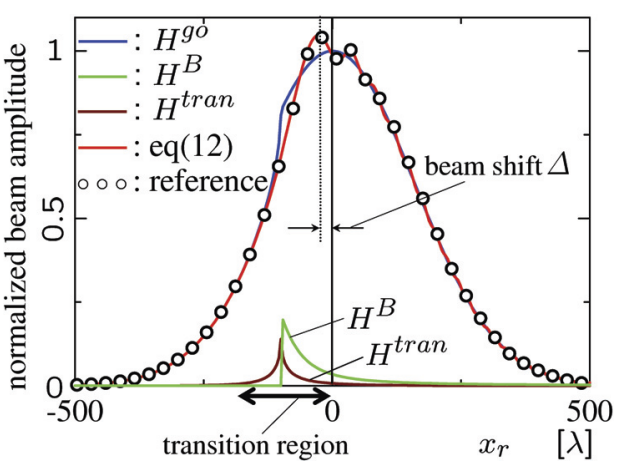

(b) $\theta_{b}=60.7^{\circ}$

Fig. 3. Numerical calculations of Gaussian beams reflection for TM case $\left(m=n^{2}\right)$. Numerical parameters: $h=2000 \lambda, z_{b}=2000 \lambda, W=10 \lambda$, $\varepsilon_{1}=1.333 \varepsilon_{0}, \varepsilon_{2}=1.0 \varepsilon_{0}, n=0.866$, and $\delta=60^{\circ}$.

\section{Conclusion}

We have derived the novel uniform asymptotic solution for the reflection of the Gaussian beam that is incident from a denser dielectric medium. The validity of the novel uniform asymptotic solution has been confirmed by comparing with the reference solution obtained numerically. It has been shown 
that the transition beam plays an important role in the transition region. It becomes apparent that the Gaussian beam is shifted to either direction near the critical angle of the total reflection by the interference of the geometrically reflected beam and the lateral beam.

\section{Acknowledgment}

This work was supported in part by the Grant-in-Aid for Scientific Research (C) (21560424) from Japan Society for the Promotion of Science (JSPS).

The authors would like to thank Mr. H. Yamada for his helpful comments and previous work. 